\title{
ENLIGHT: THE EUROPEAN NETWORK FOR LIGHT ION HADRON THERAPY
}

\author{
Manjit Dosanjh, Manuela Cirilli, Virginia Greco, and Annelie E. Meijer*
}

\begin{abstract}
The European Network for Light Ion Hadron Therapy (ENLIGHT) was established in 2002 to coordinate European efforts on hadron therapy (radiotherapy performed with protons and light ions instead of high-energy photons). The ENLIGHT network is formed by the European Hadron Therapy Community, with more than 300 participants from 20 different countries. A major success of ENLIGHT has been uniting traditionally separate communities so that clinicians, physicists, biologists, and engineers with experience and interest in particle therapy work together. ENLIGHT has been a successful initiative in forming a common European platform and bringing together people from diverse disciplines. ENLIGHT demonstrates the advantages of regular and organized exchanges of data, information, and best practices, as well as determining and following strategies for future needs in research and technological development in the hadron therapy field.

Health Phys. 103(5):674-680; 2012
\end{abstract}

Key words: National Council on Radiation Protection and Measurements; medical radiation; protons; radiation therapy

\section{INTRODUCTION}

CANCER IS a major problem of modern society, and radiation therapy plays an essential role in its treatment. The main aim of radiation therapy is to deliver a maximally effective dose of radiation to a designated tumor site while sparing the surrounding healthy tissues as much as possible. Conventional $\mathrm{x}$-ray radiation therapy is characterized by almost exponential attenuation and absorption and consequently delivers the maximum energy near the beam entrance, but it continues to deposit significant energy at distances beyond the cancer target. To compensate for the disadvantageous depth-dose characteristics of $\mathrm{x}$-rays and to better conform the radiation dose distribution to the shape of many cancers, the radiation oncologists use complex

*CERN, European Organization for Nuclear Research, 1211 Geneva 23, Switzerland.

The authors declare no conflict of interest.

For correspondence contact: Manjit Dosanjh at CERN, European Organization for Nuclear Research, 1211 Geneva 23, Switzerland, or email at manjit.dosanjh@cern.ch.

(Manuscript accepted 18 May 2012)

0017-9078/12/0

Copyright $(\underset{2}{2012}$ Health Physics Society

DOI: 10.1097/HP.0b013e3182606520 conformal and intensity-modulated radiation therapy techniques. These involve the use of computerized treatment plan optimization tools, thus achieving a better dose conformity and minimizing the total energy deposition to the normal tissues.

Robert Wilson, physicist and founder of Fermilab, proposed the use of hadrons for cancer treatment in 1946 (Wilson 1946). This idea was first applied at the Lawrence Berkeley Laboratory, where 30 patients were treated with protons between 1954-1957 (Tobias et al. 1958). While at the beginning only protons where used, later the idea was extended to ions (atoms deprived of one or more electrons, which consequently have a net electromagnetic charge), in particular carbon ions. The name hadron therapy is then used for ions as well, even if, strictly speaking, hadrons are considered the only subatomic particles composed of quarks (such as protons and neutrons). It is also worth mentioning that the ions employed, as the carbon ions, are defined as "light" by physicists, while they are considered "heavy" by radiobiologists.

Radiation therapy with such charged particles (protons and light ions) offers several advantages. The heavier charged hadrons penetrate the patient with minimal lateral diffusion and deposit their maximum dose at the end of their range, effectively sparing surrounding tissues. The cancer dose profile can be precisely shaped using narrow focused and scanned pencil beams of variable penetration depth (Schulz-Ertner and Tsujii 2007).

Protons have similar biological effects as $\mathrm{x}$-rays but different depth-dose profiles, with the highest dose at the end of their range. Thus, in some cases, lower tissue damage can be obtained than by using $\mathrm{x}$-rays. Protons have a biological effect around 10\% higher than x-rays but deposit less energy in healthy tissues. Thus with protons, a higher tumor control probability can be obtained if dose is increased, while allowing reduced complications in a larger volume of normal surrounding tissue (Lomax et al. 2004; Delaney and Kooy 2008).

Carbon ions deposit about 24 times more energy in a cell than protons having the same range. In the last centimeters of the range, the linear energy transfer (LET) is 
much larger than that of x-rays and protons (low-LET radiations). The resulting DNA damage includes more complex double-strand breaks and lethal chromosomal aberrations, which are less efficiently repaired by the normal cellular mechanisms. From the entrance point to about $5 \mathrm{~cm}$ before the end of the range in tissue, carbon ions deposit lower energy densities behaving as low-LET radiations, and the DNA damage is more repairable. The effects produced at the end of the range are qualitatively different from those produced by the other classes of radiations and open the way to a strategy to overcome radioresistance, often due to hypoxia of the tumor cells. For these reasons, carbon ions with their higher relative biological effectiveness (RBE) at the end of their range (at around a factor of three) can control tumors that are normally resistant to $\mathrm{x}$-rays and possibly protons (Amaldi and Kraft 2007; Jones 2008).

Europe has played and continues to play a key role in the development of hadron therapy. In 1997, for the first time, treatments with actively scanned carbon ions were performed at the Gesellschaft für Schwerionenforschung (GSI) Center in Germany (Kraft 1998; Amaldi and Kraft 2007).

The European Network for Light Ion Hadron Therapy (ENLIGHT 2002), which had its inaugural meeting at the European Organization for Nuclear Research (CERN) in February 2002, was established to coordinate European efforts in using light-ion beams for radiation therapy (Dosanjh et al. 2007). Funded by the European Commission (EC) for $3 \mathrm{y}$, the network was formed from a collaboration of European centers, institutions, and scientists, all involved in research and in the promotion and realization of hadron therapy facilities in Europe.

The ENLIGHT network has been instrumental in bringing together different European centers to promote hadron therapy and to help establish international discussions comparing the respective advantages of intensitymodulated radiation proton and carbon therapies. A major success of ENLIGHT has been the creation of a multidisciplinary platform bringing together communities that were traditionally separated, so that clinicians, physicists, biologists, and engineers work side-by-side.

In 2006, a brainstorming session amongst clinicians, oncologists, physicists, radiobiologists, information and communication technology experts, and engineers from around 20 European countries took place at CERN. The community felt that ENLIGHT was a key ingredient for future progress and therefore should be maintained and broadened. The main objective of this enhanced collaboration, called ENLIGHT++, is to form a consensus from representatives of different disciplines and national programs on what is most beneficial for the patient. It was also agreed that this goal could be met by reinforcing the existing pan-European network and focusing on two com- plementary aspects: the "research" in areas needed for effective hadron therapy and the "networking" needed for establishing and implementing common standards and protocols for treating patients.

The ENLIGHT++ network meets the need for coordinating and reinforcing the research activities for the improvement of hadron therapy technology at the European centers that are currently under construction and for the development of new facilities. It provides a common European platform for fostering and coordinating collaborations between national research activities, such as development of accelerators, detectors, image reconstruction and processing, radiobiology, oncology, and clinical research.

In the past few years, Europe has made important steps in the development and construction of hospitalbased "dual" centers for carbon ions and protons. In particular, the Heidelberg Ion Radiation Therapy Center in Germany treated its first patient in November 2009 (Combs et al. 2010), and the Italian Centro Nazionale di Adroterapia Oncologica in Pavia started treating patients in September 2011 (Orrechia et al. 2009). However, the challenges posed by the size and cost of hadron therapy centers are numerous, and the design of optimal and standardized facilities must necessarily come from a global, interdisciplinary, and transnational research effort. Several Asian countries, in particular Japan but also Korea and China, are interested in hospital-based hadron therapy centers (Schulz-Ertner and Tsujii 2007).

Under the umbrella of ENLIGHT, there are currently four EC funded projects (Dosanjh et al. 2010): Particle Training Network for European Radiotherapy (PARTNER), Union of Light Ion Centers in Europe (ULICE 2009), European Novel Imaging System for Ion Therapy (ENVISION), and Research Training in Three-Dimensional Digital Imaging for Cancer Radiation Therapy (ENTERVISION). All these projects are directed toward the various aspects of developing, establishing, and optimizing hadron therapy (ENVISION 2008).

The initiatives involve integrating clinical, biological, and technical knowledge as well as training the future generation at a Europe-wide level, so that hadron therapy becomes widely available for the benefit of all European inhabitants. Specifically it aims at:

- identifying the critical topics and focusing the research on key areas in order to define and develop particle therapy and extend its benefits throughout Europe and eventually worldwide, complementary to other treatments;

- developing a common European platform to validate the efficacy of hadron therapy, starting first with the most advanced facilities in Heidelberg and Pavia; 
- developing the technical expertise and widespread knowledge for a therapeutic use of particle therapy and creating the appropriate professional knowledge needed for European-wide expansion;

- enhancing the cost effectiveness of hadron therapy by improving quality of life and reducing the overall cost of treatment;

- integrating hadron therapy within the best available multi-disciplinary management of cancer treatment; and

- training the future scientists needed for this emerging field.

This article focuses on two concrete examples, radiobiology and medical imaging, to illustrate the role of ENLIGHT in research and development connected to hadron therapy. It also shows how this international and multidisciplinary network promotes training for future generations of scientists, which is essential to operate the new and upcoming treatment facilities.

\section{TRAINING}

With cancer continuing to be an increasing health risk, Europe faces a major challenge of training more competent researchers in this field. In this decade, hadron therapy is growing rapidly, and Europe has made important steps in the development and construction of hospitalbased dual centers for carbon ions and protons.

There is an urgent need for qualified researchers from a range of disciplines to work on translational research for hadron therapy. Already the existing centers in Europe are running at less than maximum capacity (both with respect to clinical services and research and development), and they would need to increase patient throughput. Many of the facilities are limited by their manpower, and recruitment is not easy due to lack of experienced/trained personnel. Even industries in ion beam therapy are limited in their capacities for delivery and installation, mainly due to manpower restrictions. There is a clear need and European added value for training the future researchers and personnel.

ENVISION (2008) has been addressing the imperative need for highly trained scientists since 2008, with the establishment of PARTNER (2008), which is a Marie Curie Initial Training Network involving 10 institutes and research centers and two commercial partners in Europe. It is coordinated by CERN and composed of 20 early stage researchers and five experienced researchers from 15 different nationalities. This project represents the first organic attempt at multidisciplinary training of scientists in this rapidly growing and diverse field. It is important that all the researchers learn, or at least be exposed to, as many aspects of this newly emerging multidisciplinary field as possible.
PARTNER covers nine different project objectives:

1. clinical and epidemiology studies and patient selection;

2. radiobiology;

3. treatment planning;

4. dosimetry simulation with Fluktuating Kaskade (FLUKA) and GEometry ANd Tracking (GEANT);

5. image guided hadron therapy;

6. positron emission tomography (PET) prototype for in situ monitoring;

7. novel gantry design;

8. information and communication technologies and networking in hadron therapy; and

9. novel accelerator machine study.

In September 2010, the PARTNER mid-term review took place, and the EC representatives were highly impressed with the progress of the project, recommending that the community propose a follow-up on project.

A second Marie Curie Initial Training Network, ENTERVISION (2011), was launched by the ENLIGHT community in February 2011. The project brings together 10 academic institutes and research centers, as well as the two leading European companies in particle therapy, and is also coordinated by CERN. ENTERVISION was established in response to a critical need to reinforce research in online three-dimensional digital imaging and to train professionals in order to deliver some of the key elements for early detection and more precise treatment of tumors. The 12 early-stage and four experienced researchers will join a dynamic program and will become part of a network of experts with a unique set of competencies, expertise, infrastructures, and training possibilities.

ENTERVISION will rely on another EC-funded project under the ENLIGHT umbrella, called ENVISION, to provide the hands-on work in the following fields:

- development of in-beam PET monitoring techniques;

- development of single particle tomography techniques;

- adaptive treatment planning and organ motion;

- optical imaging, cell irradiation, and biological phantom design; and

- Monte Carlo simulation of in vivo dosimetry.

The main benefits resulting from the ENLIGHT training program will be:

- creation of a Europe-wide community of researchers and experts who, although working in different European countries, have the same background and knowledge;

- enabling European researchers and European industry to keep their leading position in ion therapy; and

- moving the field forward. 


\section{RESEARCH NEEDS IN RADIATION BIOLOGY}

In 1956, Puck and Marcus (1956) developed the clonogenic cell survival assay for in vitro studies and measurements of toxicity of different damaging agents. Today this survival assay is considered as the "gold standard" for measuring the cell-killing effects of ionizing radiation in different biological systems (Blakely 1992).

Since the 1970s, biological experiments have been performed both in vitro and in vivo using different ions, LET, doses, cell types, and organisms. It is well known that charged particles with high-LET induce cellular damage that is more severe compared to damage induced by lowLET (Pouget and Mather 2001). When an ion traverses biological matter, like a cell, it deposits energy in clusters. These patterns of clusters are often called "track structures" and are both LET- and ion-dependent: the higher the LET and the heavier the ion, the more complex are the cluster patterns. It is also known that the effects of oxygen are less important for cell killing for high-LET compared to low-LET and that the oxygen enhanced ratio (OER) for high-LET is close to one. This is an additional reason why radiation-resistant tumors respond better to hadron therapy than conventional radiation treatment (Hall and Giaccia 2006).

Although much is known about the biological effects of different ions, a detailed account of how LET, OER, and RBE change as a function of the ion species, the incident beam energy, and the depth in the tissue still needs to be studied. One of the reasons is the growing interest and development of hadron therapy not only in Europe but also worldwide. Information of the RBE-LET and the RBEOER relations is important and should be included in treatment planning with ions. Also, further investigations are needed to clarify why and how charged-particle beams have a greater effect than other radiation therapies. Even though protons and carbon ions are used clinically with great success, there are further needs to get the behavior of these beams adequately characterized. In addition, it is important to study other ions as well that might be more optimal than protons and carbon ions to be used in the clinic. There are suggestions that in addition to carbon ions, other so-called light ions such as lithium, boron, nitrogen, and oxygen could be of interest for hadron therapy (Brahme 2010). So far there are limited reports of the biological effects of light ions, and it is of greatest interest to investigate the biological effects of these ions as well as obtain further information on the beam fragmentation that is needed for optimization of light ion hadron therapy.

Enhanced understanding of the mechanisms behind the biological response of cell killing is key to improving the effectiveness of tumor therapy and also to reducing possible side effects in normal tissues. Therefore, future radiation biology studies should focus not only on lethality but also on other functional cell inactivation endpoints and molecular and genetic effects (Blakely 1992).

Some of this much needed research is included in the radiobiology projects of PARTNER. There are three main radiobiology projects in PARTNER, including studies on effects of different ions with regard to:

- RBE-LET and RBE-OER relations;

- combination effects of chemotherapeutic drugs; and

- underlying cellular and genetic responses.

These projects are represented by five ESRs and work packages at three different PARTNER institutes (i.e., GSI, Darmstadt, Germany, and Karolinska Institute, Stockholm, Sweden, as well as the University of Surrey, United Kingdom, and in collaboration with the Heavy Ion Medical Accelerator in Chiba, Japan, and the University of Cambridge, United Kingdom).

PARTNER covers studies on the RBE-LET relations of different ions including both lighter (lithium, boron, carbon, and nitrogen) but also heavier (argon and iron) ones. Both normal cells and tumor cell lines and strains of different origin and gene status are being investigated. Tumor cells that are of most interest to study are those that are specifically known to be radioresistant to conventional radiation and chemotherapy (e.g., lung, prostate, melanoma, glioblastoma, and head and neck cells). Within PARTNER, studies on the RBE-OER relation are also performed and include investigations during both acute and chronic anoxic and hypoxic conditions. Different mathematical modeling techniques are used and compared for the different doseresponse data settings that can be employed and benefit future treatment planning.

The concept of RBE for different biological endpoints than for the classical clonogenic cell survival assay is not always easily performed and calculated. In PARTNER, these kinds of studies are being carried out, and different modes of cell inactivation pathways (e.g., apoptosis, necrosis, senescence, formation of micronuclei, and cell-cycle arrest) are investigated in parallel with the use of different techniques. Apoptosis is one of the preferable types of pathway to kill a cell, since these cells will be removed in vivo without negatively affecting the surrounding tissue, whereas necrosis leads to inflammation. Senescence (i.e., permanent cell-cycle arrest) is also a preferable type of cell inactivation when it comes to inactivating a tumor. Within the PARTNER projects, studies like these are ongoing, and also targeted experiments using microbeams are planned for more specific target signaling and track structure studies.

Glioblastomas are radioresistant, and radiotherapy treatments combined with chemotherapeutic radiosensitizing drugs are also a promising field of hadron therapy for 
treatment of these tumors. There are some clinical trials using the radiosensitizing drug temosolomide (Stupp et al. 2005). However, there are further needs for these kinds of studies in vitro using not only carbon ions but also other lighter ions. This is one of the projects being investigated in PARTNER that could improve the treatment of radioresistant tumors.

The knowledge that the results from the different PARTNER projects in radiation biology will benefit the understanding of how different ions affect different cellular systems both on the cellular and molecular level. This is important for the optimization of hadron therapy to give a more specific treatment for each individual patient to be treated.

\section{DEVELOPMENT OF NOVEL MEDICAL IMAGING TOOLS}

Medical imaging tools are essential not only for better diagnosis but also for optimal treatment. This is particularly true in the case of hadron therapy, where real-time monitoring of the delivered dose and assessment of the tumor volume are key to improving the results of the treatment and to minimizing damage to healthy tissues.

In fact, active scanning techniques allow defining the shape and size of the tumor target in three dimensions and irradiating it with pencil beams of protons or ions. To be successful, the irradiation must adequately cover the cancer volume while sparing as much as possible of the surrounding healthy tissues. The conventional methods used for the assessment of patient positioning in all x-ray-based radiation therapy procedures are not applicable in hadron therapy. X-ray imaging techniques rely on the fact that a non-negligible fraction of the beam traverses the patient, thus allowing the therapist to simply reconstruct the patient position from the bony anatomy or fiducial markers. In the case of hadrons, the dose distribution follows the Bragg peak and sharply decreases behind the target, with almost no exit dose (for protons) or a very small one (for carbon and other light ions).

Novel imaging modalities are therefore needed to fully exploit the potential of hadron therapy for cancer treatment. ENLIGHT addresses the crucial topics of in vivo monitoring of the delivered dose and quality assurance for hadron therapy with the EU-funded project ENVISION, which started in February 2010. The project gathers 16 leading European research centers and industrial partners and is coordinated by CERN.

ENVISION aims at developing innovative solutions for real-time noninvasive monitoring, quantitative imaging, precise determination of delivered dose, fast feedback for optimal treatment planning, real-time response to moving organs, and simulation studies.
The project will tackle the improvement of the quality of in-beam PET (ibPET) (Shakirin et al. 2007) images in two different ways: the time-of-flight (TOF) and the single particle tomography (ibSPAT) approaches.

TOF techniques limit the region of interest by measuring the time difference between the two co-linear gamma rays. The imaging power of the method depends on the accuracy of the TOF determination: the latest generation of commercial PET scanners has typical resolution of the order of $500 \mathrm{ps}$, which translates into a region of interest of about $15 \mathrm{~cm}$ and is in general larger than the volume to irradiate. ENVISION will assess the improvements in TOF determination achievable with two alternative technologies, fast scintillating crystals and resistive plate chambers, which can potentially achieve a resolution of a 100 ps or better, corresponding to a spatial resolution below $2 \mathrm{~cm}$ (Cerron Zeballos et al. 1996; Lecoq 1999).

Single-particle tomography is a novel method of in vivo dosimetry based on the detection of prompt radiation components (gamma rays or light-charged particles) following nuclear reactions between the particles of the therapeutic beam and the atomic nuclei of the tissue. The ibSPAT technique has the capability to overcome the inherent sensitivity of ibPET to metabolism, as the spatial distribution of prompt radiation components is not influenced by metabolic processes. Within ENVISION, the potential of prompt particle detection for therapy quality assurance will be thoroughly investigated for the first time. Single photon emission tomography is already widely applied in nuclear medicine and therefore has a superior potential for in vivo therapy monitoring with respect to light charged particle identification. However, the devices used in nuclear medicine are optimized for monoenergetic photons of rather low energy, while the prompt photons emitted during ion-beam irradiation have quite a broad energy spectrum (between 0 and $10 \mathrm{MeV}$ ). This implies that novel imaging devices will have to be developed during the course of the project (Testa et al. 2008). Light charged particles from nuclear interactions do not pose special challenging of the detectors. On the other hand, they do require careful studies to determine whether they can actually carry relevant dosimetric information, as a considerable fraction of these particles will be absorbed in the patient's body and in the surrounding equipment, and they are not emitted isotropically.

ENVISION will also apply in vivo ibPET and ibSPAT imaging techniques (Parodi et al. 2008) to the monitoring of moving organs for the first time. Currently, information on organ motion can be assessed prior to the treatment on the basis of time-resolved four-dimensional computedtomography data and then monitored during the treatment, typically using an external surrogate. Validation of the correlation between the external surrogate and internal organ 
motion may be updated during treatment using fluoroscopy or other imaging modalities (e.g., ultrasound). There is no commercially available technique capable of monitoring the actual interaction of the beam with the tissue and hence of evaluating deviations between the planned and delivered dose. ENVISION will therefore strive to improve the quality and reliability of hadron therapy for moving targets by assessing the feasibility and potential of in vivo dosimetric systems for the analysis of motioncompensated dose delivery and by optimizing their imaging performance.

An important focus of ENVISION is the development of techniques for fast and automatic integration of dosimetric information from ibPET, ibSPAT, and fourdimensional monitoring into the treatment planning and delivery workflow. Such developments are essential for the timely discovery of treatment delivery errors and for adaptive radiotherapy, where the therapeutic irradiation plan is adapted continuously to account for changes in patient anatomy, tumor size, and tissue dosimetry.

ENVISION also engages in the development of advanced Monte Carlo simulations for in vivo dosimetry (Agostinelli et al. 2003; Buvat and Lazaro 2006; Battistoni et al. 2007). In fact, simulation tools are needed to estimate accurately the real dose distribution from the signal obtained by PET or single photon emission tomography monitoring. The project will review the available models and experimental data with the aim of identifying possible shortcomings and of proposing an experimental program for the measurement of the relevant physical processes. In addition, ENVISION will provide efficient and validated computational tools that will enable full and accurate modeling of in-beam PET experiments. In parallel, fast simulation tools for online use in a treatment planning system will be developed.

Finally, ENVISION will provide a platform for the training of future generations of researchers under the umbrella of the newly funded ENTERVISION project.

\section{CONCLUSION}

The European scientific community in hadron therapy research has grown rapidly over the last $10 \mathrm{y}$. The ENLIGHT network was a first successful approach toward a common European platform for multi-center and multidisciplinary integration of the European research community. ENLIGHT continues to enable the community interested in hadron therapy to participate in and contribute actively to the further development of this rapidly growing field in a systematic way. A portfolio of training and research projects sponsored by ENLIGHT is helping to establish hadron therapy as a scientifically demonstrated, clinically recognized, technically mature, and affordable cancer treatment modality in Europe. ENLIGHT's key vision was and continues to be the promotion and the optimization of hadron therapy for cancer treatment at a panEuropean level.

Acknowledgments - PARTNER is a Marie Curie International Training Network funded by the European Commission under Grant Agreement N. 215840 (http://partner.web.cern.ch/PARTNER). ULICE is co-funded by the European Commission within the Framework Programme 7 Capacities Specific Programme, under Grant Agreement N. 228436 (http://ulice.web. cern.ch/ULICE). ENVISION is co-funded by the European Commission within the Framework Programme 7 Cooperation Specific Programme under Grant Agreement N. 241851 (http://envision.web.cern.ch/ENVISION). ENTERVISION is a Marie Curie International Training Network funded by the European Commission under Grant Agreement N. 264552 (http://entervision. web.cern.ch/ENTERVISION). A. E. Meijer is additionally affiliated at the Medical Radiation Physics, Department of Oncology-Pathology, Karolinska Institutet, P.O. Box 260, SE-171 76 Stockholm, Sweden, and at the International Open Laboratory, National Institute of Radiological Sciences, 4-9-1 Anagawa, Inage-ku, Chiba 263-8555, Japan.

\section{REFERENCES}

Agostinelli S, Allison J, Amako K, Apostolakis J, Araujo H, Arce P, Asai M, Axen D, Banerjee S, Barrand G, Behner F, Bellagamba L, Boudreau J, Broglia L, Brunengo A, Burkhardt H, Chauvie S, Chuma J, Chytracek R, Cooperman G, Cosmo G, Degtyarenko P, Dell'Acqua A, Depaola G, Dietrich D, Enami R, Feliciello A, Ferguson C, Fesefeldt H, Folger G, Foppiano F, Fortia A, Garelli S, Giani S, Giannitrapani R, Gibin D, Gomez Cadenas JJ, Gonzalez I, Gracia Abril G, Greeniaus G, Greiner W, Grichine V, Grossheim A, Guatelli S, Gumplinger P, Hamatsu R, Hashimoto K, Hasui H, Heikkinen A, Howard A, Ivanchenko V, Johnson, A, Jones FW, Kallenbach J, Kanaya N, Kawabata M, Kawabata Y, Kawaguti M, Kelner S, Kent P, Kimura A, Kodama T, Kokoulin R, Kossov M, Kurashige H, Lamanna E, Lampen T, Lara V, Lefebure V, Leib F, Liendl, M, Lockman W, Longo F, Magni S, Maire M, Medernach E, Minamimoto K, Mora de Freitas P, Morita Y, Murakami K, Nagamatu M, Nartallo R, Nieminen P, Nishimura T, Ohtsubo K, Okamura M, O’Neal S, Oohata Y, Paech K, Perl J, Pfeiffer A, Pia MG, Ranjard F, Rybin A, Sadilov S, Di Salvo E, Santin G, Sasaki T, Savvas N, Sawada Y, Scherer S, Sei S, Sirotenko V, Smith D, Starkov N, Stoecker H, Sulkimo J, Takahata M, Tanaka S, Tcherniaev E, Safai Tehrani E, Tropeano M, Truscott, P, Uno H, Urban L, Urban P, Verderi M, Walkden A, Wander W, Weber H, Wellisch JP, Wenaus T, Williams DC, Wright D, Yamada T, Yoshida H, Zschiesche D. Geant4, a simulation toolkit. Nucl Instr Meth Phys Res A 506:250-303; 2003.

Amaldi U, Kraft G. European developments in radiotherapy with beams of large radiobiological effectiveness. J Radiat Res 48:A27-A41; 2007.

Battistoni G, Muraro S, Sala P.R, Cerutti F, Ferrari A, Roesler S, Fasso A, Ranft J. FLUKA: a multi-particle transport code. In: Albrow M, Raja R, eds. Proceedings of the Hadronic Shower Simulation Workshop 2006. AIP Conference Proceeding 896; 2007: 31-9.

Blakely E. Cell inactivation by heavy charged particles. Radiat Environ Biophys 31:181-96; 1992.

Brahme A. Plans for ion radiation therapy at the Karolinska Institute and University Hospital. In: Proceedings of an International Japanese-European joint symposium on ion cancer therapy and NIRS-KI joint symposium on ion-radiation sciences. Stockholm: Karolinska Institutet; NIRS-M235; 2010: 5-10. 
Buvat I, Lazaro D. Monte Carlo simulations in emission tomography and GATE: an overview. Nucl Instr Meth Phys Res 569:323-329; 2006.

Cerron Zeballos E, Crotty I, Hatzifotiadou D, Lamas Valverde J, Neupane S, Williams MCS, Zichichi A. A new type of resistive plate chamber: the multigap RPC. Nucl Instr Meth A374:132-135; 1996.

Combs S, Jakel O, Haberer T, Debus J. Particle therapy at the Heidelberg Ion Therapy Center (HIT) — integrated researchdriven university-hospital-based radiation oncology service in Heidelberg, Germany. Radiother Oncol 95:41-44; 2010.

Delaney T, Kooy H. Proton and charged particle radiotherapy. Philadelphia: Lippincott Williams and Wilkins; 2008.

Dosanjh MK, Hoffmann HF, Magrin G. Status of hadron therapy in Europe and the role of ENLIGHT. Nucl Instrum Meth Phys Res A 571:191-194; 2007.

Dosanjh M, Jones B, Mayer R. ENLIGHT and other EU-funded projects in hadron therapy. Br J Radiol 83:811-813; 2010.

ENLIGHT 2002. Available at http://cern.ch/ENLIGHT. Accessed 19 July 2011.

ENTERVISION 2011. Available at http://entervision.web.cern.ch/ ENTERVISION. Accessed 19 July 2011.

ENVISION 2008. Available at http://envision.web.cern.ch/ ENVISION. Accessed 19 July 2011.

Hall EJ, Giaccia AJ. Radiobiology for the radiobiologist. New York: Lippincott Williams and Wilkins; 2006.

Jones B. The potential advantages of charged particle radiotherapy using protons or light ions. Clin Oncol 20:555-563; 2008.

Kraft G. Radiotherapy with heavy ions: radiobiology, clinical indications and experience at GSI, Darmstadt. Tumori 84: 200-204; 1998.

Lecoq P. New scintillating crystals for medical imaging. In: Proceedings of CALOR99: calorimetry in high energy physics. Lisbon, Portugal: World Scientific; 1999: 667-674.

Lomax A, Pedroni E, Rutz H, Goitein G. The clinical potential of intensity modulated proton therapy. Z Med Phys 14: 147-52; 2004.

Orrechia R, Fossati P, Rossi S. The National Center for Oncological Hadron Therapy: status of the project and future clinical use of the facility. Tumori 95:169-176; 2009.
Parodi K, Bortfeld T, Enghardt W, Fiedler F, Knopf A, Paganetti H, Pawelke J, Shakirin G, Shih H. PET imaging for treatment verification of ion therapy: implementation and experience at GSI Darmstadt and MGH Boston. Nucl Instrum Methods Phys Res 591:282-286; 2008.

PARTNER. 2008. Available at http://partner.web.cern.ch/ PARTNER. Accessed 19 July 2011.

Pouget JP, Mather SJ. General aspects of the cellular response to low- and high-LET radiation. Euro J Nucl Med 28: 541-561; 2001.

Puck TT, Markus PI. Action of x-rays on mammalian cells. J Exp Med 103:653-666; 1956.

Schultz-Ertner D, Tsujii H. Particle radiation therapy using protons and heavier ion beams. J Clin Oncol 25:953-964; 2007.

Shakirin G, Crespo P, Braess H, Enghardt W. Influence of the time of flight information on the reconstruction of in-beam PET data. In: IEEE Nuclear Science Symposium conference record. Honolulu, HI: IEEE; 2007; 379.

Stupp R, Mason WP, van den Bent MJ, Weller M, Fisher B, Taphoorn MJB, Belanger K, Brandes AA, Marosi C, Bogdahn U, Curschmann J, Janzer RC, Ludwin SK, Gorlia T, Allgeier A, Lacombe D, Cairncross JG, Eisenhauer E, Mirimanoff RO. Radiotherapy plus concomitant and adjuvant temazolomide for glioblastoma. N Engl J Med 352:987-996; 2005.

Testa E, Bajard M, Chevallier M, Dauvergne D, Le Foulher F, Freud N, Letang J-M, Poizat J-C, Ray C, Testa M. Monitoring the Bragg peak location of $73 \mathrm{MeV} / \mathrm{u}$ carbon ions by means of prompt gamma-ray measurements. Appl Phys Lett 93: 93503-93506; 2008.

Tobias CA, Lawrence JH, Born JL, Mc Combs CR, Roberts JE, Anger HO, Low-Beer BV, Huggins CB. Pituitary irradiation with high-energy proton beams: a preliminary report. Cancer Res 18:121-134; 1958.

ULICE. 2009. Available at http://ulice.web.cern.ch/ULICE. Accessed 19 July 2011.

Wilson R. Radiological use of fast protons. Radiol 47: 487-491; 1946. 